\title{
Dependence of nuclear magnetic moments on quark masses and limits on temporal variation of fundamental constants from atomic clock experiments
}

\author{
V.V. Flambaum, A.F. Tedesco \\ School of Physics, The University of New South Wales, Sydney NSW 2052, Australia
}

(Dated: July 18, 2018)

\begin{abstract}
We calculate the dependence of the nuclear magnetic moments on the quark masses including the spin-spin interaction effects and obtain limits on the variation of the fine structure constant $\alpha$ and $\left(m_{q} / \Lambda_{Q C D}\right)$ using recent atomic clock experiments examining hyperfine transitions in $\mathrm{H}, \mathrm{Rb}, \mathrm{Cs}$, $\mathrm{Yb}^{+}$and $\mathrm{Hg}^{+}$and the optical transition in $\mathrm{H}, \mathrm{Hg}^{+}$and $\mathrm{Yb}^{+}$.

PACS numbers: 06.20.Jr, 06.30.Ft, 21.10.Ky
\end{abstract}

\section{INTRODUCTION}

Theories unifying gravity with other interactions suggest a possibility of temporal and spatial variation of the fundamental constants of nature (see e.g. review [1] where the theoretical models and results of measurements are presented). There are hints for the variation of the fundamental constants in Big Bang nucleosynthesis, quasar absorption spectra and Oklo natural nuclear reactor data. However, a majority of publications report only limits on possible variations. For example, comparison of different atomic clocks gives limits on present time variation of the fundamental constants.

A large fraction of the publications discuss the variation of the fine structure constant $\alpha=e^{2} / \hbar c$. The hypothetical unification of all interactions implies that a variation in $\alpha$ should be accompanied by a variation of the strong interaction strength and the fundamental masses. For example, the grand unification model discussed in Ref. 2] predicts the quantum chromodynamics (QCD) scale $\Lambda_{Q C D}$ (defined as the position of the Landau pole in the logarithm for the running strong coupling constant) is modified as $\delta \Lambda_{Q C D} / \Lambda_{Q C D} \approx 34 \delta \alpha / \alpha$. The variation of quark and electron masses in this model is given by $\delta m / m \sim 70 \delta \alpha / \alpha$, giving an estimate of the variation for the dimensionless ratio

$$
\frac{\delta\left(m_{q} / \Lambda_{Q C D}\right)}{\left(m_{q} / \Lambda_{Q C D}\right)} \sim 35 \frac{\delta \alpha}{\alpha}
$$

The coefficient here is model dependent but large values are generic for grand unification models in which modifications come from high energy scales; they appear because the running strong-coupling constant and Higgs constants (related to mass) run faster than $\alpha$. If these models are correct, the variation in quark masses and the strong interaction scale may be easier to detect than a variation in $\alpha$.

One can only measure the variation of dimensionless quantities. We want to extract from the measurements the variation of the dimensionless ratio $m_{q} / \Lambda_{Q C D}$ where $m_{q}$ is the quark mass (with the dependence on the renormalization point removed). A number of limits on the variation of $m_{q} / \Lambda_{Q C D}$ have been obtained recently from consideration of Big Band nucleosynthesis, quasar ab- sorption spectra and the Oklo natural nuclear reactor, which was active about 1.8 billion years ago [3, 4, [5].

Karshenboim [6] has pointed out that measurements of ratios of hyperfine structure intervals in different atoms are sensitive to variations in nuclear magnetic moments. However, the magnetic moments are not the fundamental parameters and can not be directly compared with any theory of the variations. Atomic and nuclear calculations are needed for the interpretation of the measurements. Below, we calculate the dependence of nuclear magnetic moments on $m_{q} / \Lambda_{Q C D}$ by building on recent work and incorporating the effect of the spin-spin interaction between nucleons. We obtain limits on the variation of $m_{q} / \Lambda_{Q C D}$ from recent experiments that have measured the time dependence of the ratios of the hyperfine structure intervals of ${ }^{133} \mathrm{Cs}$ and ${ }^{87} \mathrm{Rb}$ [8], ${ }^{133} \mathrm{Cs}$ and ${ }^{1} \mathrm{H}$ [9], ${ }^{171} \mathrm{Yb}^{+}$and ${ }^{133} \mathrm{Cs}$ [10], ${ }^{199} \mathrm{Hg}^{+}$and ${ }^{1} \mathrm{H}$ [11], the ratio of the optical frequency in ${ }^{199} \mathrm{Hg}^{+}$to the hyperfine frequency of ${ }^{133} \mathrm{Cs}$ [12], the ratio of the optical frequency in ${ }^{1} \mathrm{H}$ to the hyperfine frequency of ${ }^{133} \mathrm{Cs}$ [13], and the ratio of the optical frequency in ${ }^{171} \mathrm{Yb}^{+}$to the hyperfine frequency of ${ }^{133} \mathrm{Cs}$ [14]. It has been suggested in Ref.[15] that the effects of the fundamental constants variation may be enhanced 2-3 orders of magnitude in diatomic molecules like LaS, LaO, LuS, LuO. Therefore, we also present the results for ${ }^{139} \mathrm{La}$.

During the calculations, we shall assume (for notational convenience) that the strong interaction scale $\Lambda_{Q C D}$ does not vary and so we shall speak about the variation of masses (this means that we measure masses in units of $\left.\Lambda_{Q C D}\right)$. We shall restore the explicit appearance of $\Lambda_{Q C D}$ in the final answers.

The hyperfine structure constant can be presented as

$$
A=\text { const } \times\left(\frac{m_{e} e^{4}}{\hbar^{2}}\right)\left[\alpha^{2} F_{r e l}(Z \alpha)\right]\left(\mu \frac{m_{e}}{m_{p}}\right)
$$

The factor in the first set of brackets is an atomic unit of energy. The second "electromagnetic" set of brackets determines the dependence on $\alpha$ and includes the relativistic correction factor (Casimir factor) $F_{r e l}$. The last set of brackets contains the dimensionless nuclear magnetic moment $\mu$ (that is, the nuclear magnetic moment $\left.M=\mu\left[e \hbar / 2 m_{p} c\right]\right)$ and the electron and proton masses $m_{e}$ and $m_{p}$. We might also have included a small correc- 
tion due to the finite nuclear size but its contribution is insignificant.

The ratio of two hyperfine structure constants for different atoms will cancel out some factors such as atomic unit of energy and $m_{e} / m_{p}$, and any time dependence falls on two values: the ratio of the factors $F_{\text {rel }}$ (which depends on $\alpha$ ) and the ratio of the nuclear magnetic moments (which depends on $m_{q} / \Lambda_{Q C D}$ ).

For the $F_{r e l}$ component, variation in $\alpha$ leads to the following variation of $F_{r e l}$ [11]

$$
\frac{\delta F_{r e l}}{F_{r e l}}=K_{r e l} \frac{\delta \alpha}{\alpha}
$$

and one can use the $s$-wave electron approximation for $F_{r e l}$ to get

$$
K_{r e l}=\frac{(Z \alpha)^{2}\left(12 \gamma^{2}-1\right)}{\gamma^{2}\left(4 \gamma^{2}-1\right)}
$$

where $\gamma=\sqrt{1-(Z \alpha)^{2}}$. However, numerical manybody calculations [16] give more accurate results, with a slightly higher value of $K_{r e l}$ than that given by this formula. A comparison is shown in Table \

The other component contributing to the ratio of two hyperfine structure constants is the nuclear magnetic moment $\mu$. Theoretical values for $\mu$ in the valence shell model are based on the unpaired valence nucleon and are given by Schmidt values

$$
\mu= \begin{cases}\frac{1}{2}\left[g_{s}+(2 j-1) g_{l}\right] & \text { for } j=l+\frac{1}{2} \\ \frac{j}{2(j+1)}\left[-g_{s}+(2 j+3) g_{l}\right] & \text { for } j=l-\frac{1}{2}\end{cases}
$$

The orbital gyromagnetic factors are $g_{l}=1$ for a valence proton and $g_{l}=0$ for a valence neutron. The spin gyromagnetic factors are $g_{s}\left(=g_{p}\right)=5.586$ for protons and $g_{s}\left(=g_{n}\right)=-3.826$ for neutrons. These $g$-factors depend on $m_{a} / \Lambda_{O C D}$ and previous work exploring this dependence [17, 18] is summarized below. We then use these results to consider the more realistic situation of $\mu$ having both a valence nucleon contribution and a nonvalence nucleon contribution due to the spin-spin interaction.

\section{VARIATION OF MAGNETIC MOMENT WITH VARIATION IN QUARK MASS}

\section{A. Variation in $\mu$ using valence model magnetic moment}

As a preliminary to our results and as a comparison for evaluating the effects of our calculations, we include the results of work previously done in this area [17, 18]. This work was essential to our results as the authors calculated the variation in the neutron and proton magnetic moments $\left(\mu_{n}\right.$ and $\left.\mu_{p}\right)$ with the variation in $m_{q} / \Lambda_{Q C D}$ using chiral perturbation theory.
As mentioned above, the $g$-factors depend on $m_{q} / \Lambda_{Q C D}$. The light quark mass $m_{q}=\left(m_{u}+m_{d}\right) / 2 \approx$ $5 \mathrm{MeV}$ and in the chiral limit $m_{u}=m_{d}=0$, the nucleon magnetic moment remains finite. Thus one might assume that corrections to the spin $g$-factors $g_{p}$ and $g_{n}$ are small. However, the quark mass contribution is enhanced by $\pi$-meson loop corrections to the nuclear magnetic moments, which are proportional to $\pi$-meson mass $m_{\pi} \sim \sqrt{m_{q} \Lambda_{Q C D}}$. Since $m_{\pi}=140 \mathrm{MeV}$, the contribution can be significant.

Full details of these calculations are given in Ref [17, 18]. They give the following results, which relate variations in $\mu_{n}$ and $\mu_{p}$ with variations in light and strange quark masses $\left(m_{q}\right.$ and $\left.m_{s}\right)$ :

$$
\begin{aligned}
\frac{\delta \mu_{p}}{\mu_{p}} & =-0.087 \frac{\delta m_{q}}{m_{q}} \\
\frac{\delta \mu_{p}}{\mu_{p}} & =-0.013 \frac{\delta m_{s}}{m_{s}} \\
\frac{\delta \mu_{n}}{\mu_{n}} & =-0.118 \frac{\delta m_{q}}{m_{q}} \\
\frac{\delta \mu_{n}}{\mu_{n}} & =+0.0013 \frac{\delta m_{s}}{m_{s}}
\end{aligned}
$$

Using these relations and the valence model approximations for $\mu$, we can obtain expressions of the form

$$
\frac{\delta \mu}{\mu}=\kappa_{q} \frac{\delta m_{q}}{m_{q}}+\kappa_{s} \frac{\delta m_{s}}{m_{s}}
$$

Hence for nuclei with even $\mathrm{Z}$ and a valence neutron

$$
\frac{\delta \mu}{\mu}=\frac{\delta g_{n}}{g_{n}}=-0.118 \frac{\delta m_{q}}{m_{q}}+0.0013 \frac{\delta m_{s}}{m_{s}} .
$$

For valence protons, the orbital gyromagnetic factor $g_{l}$ also has an effect. Thus for ${ }^{133} \mathrm{Cs}$ with its valence proton and $j=l-\frac{1}{2}$,

$$
\frac{\delta \mu}{\mu}=0.110 \frac{\delta m_{q}}{m_{q}}+0.016 \frac{\delta m_{s}}{m_{s}}
$$

while for ${ }^{87} \mathrm{Rb}$ with its valence proton but $j=l+\frac{1}{2}$,

$$
\frac{\delta \mu}{\mu}=-0.64 \frac{\delta m_{q}}{m_{q}}-0.010 \frac{\delta m_{s}}{m_{s}}
$$

These results can be presented using the ratio of the hyperfine constant $A$ to the atomic unit of energy $E=$ $m_{e} e^{4} / \hbar^{2}$ by defining the parameter $V$ through the relation

$$
\frac{\delta V}{V}=\frac{\delta(A / E)}{A / E} .
$$

The values for $\kappa_{q}$ and $\kappa_{s}$ in the results for $\delta \mu / \mu$ can then be combined with the corresponding values of $K_{\text {rel }}$ in Table पto give results of the form:

$$
V(M)=\alpha^{2+K_{r e l}}\left(\frac{m_{q}}{\Lambda_{Q C D}}\right)^{\kappa_{q}}\left(\frac{m_{s}}{\Lambda_{Q C D}}\right)^{\kappa_{s}} \frac{m_{e}}{m_{p}}
$$


TABLE I: Variational factor $K_{r e l}$ and $\kappa$ values for various atoms obtained using simple valence shell model (method A) as used in equation (12). The first row is given by equation (4). The second row presents the results of the more accurate many-body calculations (see ref. [16]). The numerical results marked by ${ }^{*}$ are obtained by an extrapolation from other atoms.

\begin{tabular}{c|ccccccccc}
\hline \hline Atom & ${ }^{1} \mathrm{H}$ & ${ }^{2} \mathrm{H}$ & ${ }^{3} \mathrm{He}$ & ${ }^{87} \mathrm{Rb}$ & ${ }^{111} \mathrm{Cd}$ & ${ }^{129} \mathrm{Xe}$ & ${ }^{133} \mathrm{Cs}$ & ${ }^{171} \mathrm{Yb}$ & ${ }^{199} \mathrm{Hg}$ \\
\hline$K_{\text {rel }}$ (analytical) & 0 & 0 & 0 & 0.29 & 0.53 & 0.71 & 0.74 & 1.42 & 2.18 \\
\hline$K_{\text {rel }}$ (numerical) & 0 & 0 & 0 & 0.34 & $0.6^{*}$ & $0.8^{*}$ & 0.83 & $1.5^{*}$ & 2.28 \\
\hline$\kappa_{q}$ & -0.087 & -0.020 & -0.118 & -0.064 & -0.118 & -0.118 & 0.110 & -0.118 & -0.118 \\
\hline$\kappa_{s}$ & -0.013 & -0.044 & 0.0013 & -0.010 & 0.0013 & 0.0013 & 0.016 & 0.0013 & 0.0013 \\
\hline
\end{tabular}

The calculated values of $K_{r e l}$ and $\kappa$ to be used in the expression for $\mathrm{V}(\mathrm{M})$ for various atoms are summarized in Table The factor $m_{e} / m_{p}$ will cancel out when a ratio of hyperfine transitions is used. It will, however, survive in a comparison with optical and molecular transitions.

\section{B. Variation in $\mu$ incorporating the effect of non-valence nucleons by using the experimental magnetic moment}

The results of the previous section were used to calculate the variation of $\mu$ with $m_{q} / \Lambda_{Q C D}$ based on the single particle approximation for $\mu$ (one valence nucleon) within the shell model. That is, it was assumed that the dimensionless nuclear magnetic moment is given by $\mu=g_{p}\left\langle s_{z}\right\rangle^{o}+\left\langle l_{z}\right\rangle^{0}$ for a valence proton, and $\mu=g_{n}\left\langle s_{z}\right\rangle^{o}$ for a valence neutron. Here, $g_{p}$ and $g_{n}$ are the spin gyromagnetic factors for free protons and neutrons respectively, $\left\langle l_{z}\right\rangle^{0}=j_{z}-\left\langle s_{z}\right\rangle^{o}$, and $\left\langle s_{z}\right\rangle^{o}$ is the spin expectation value of the single valence nucleon in shell model:

$$
\left\langle s_{z}\right\rangle^{o}= \begin{cases}\frac{1}{2} & \text { for } j=l+\frac{1}{2} \\ -\frac{j}{2(j+1)} & \text { for } j=l-\frac{1}{2}\end{cases}
$$

However, it is well known that this theoretical value is only an estimate of $\mu$ and the magnetic moment of the valence nucleon tends to be offset by a contribution from the core nucleons. An empirical rule is that the spin contribution of a valence nucleon should be reduced by $40 \%$ to obtain a reasonable value for the nuclear magnetic moment. This reduction may be explained by the contribution of core nucleons, which should be negative since proton and neutron magnetic moments are large and have opposite signs.

For example, a valence proton polarizes, by the spinspin interaction, core neutrons and these core neutrons give a negative contribution to the nuclear magnetic moment (polarization of the core protons by the valence proton is not important). We can estimate this offset by considering contributions to $\mu$ from the valence and core nucleons. This means we have both neutron and proton spin contributions to $\mu$ :

$$
\mu=g_{n}\left\langle s_{z_{n}}\right\rangle+g_{p}\left\langle s_{z_{p}}\right\rangle+\left\langle l_{z_{p}}\right\rangle
$$

We neglected here a small contribution of the exchange currents into the magnetic moment.

We want to evaluate the corrections to the valence model results using the very accurate experimental values of nuclear magnetic moments. Since there are three unknown parameters $\left(\left\langle s_{z_{n}}\right\rangle,\left\langle s_{z_{p}}\right\rangle,\left\langle l_{z_{p}}\right\rangle\right)$ and only one experimental value (the total magnetic moment) available, to perform an estimate we need to make approximations.

However, as we will show below, the result is not sensitive to particular approximations if we can reproduce the experimental magnetic moment exactly. Indeed, using the equations above, we can present the variation of the magnetic moment in the following form

$\delta \mu-\delta \mu^{o}=\left[0.45\left(\left\langle s_{z_{n}}\right\rangle-\left\langle s_{z_{n}}\right\rangle^{o}\right)-0.56\left(\left\langle s_{z_{p}}\right\rangle-\left\langle s_{z_{p}}\right\rangle^{o}\right)\right] \frac{\delta m_{q}}{m_{q}}$

Here $\delta \mu^{o}$ is the valence model value. For brevity we here assume that $\frac{\delta m_{q}}{m_{q}}=\frac{\delta m_{s}}{m_{s}}$ (the coefficient before $\frac{\delta m_{s}}{m_{s}}$ is small anyway)).

Let us start with the case of a valence proton. The simplest assumption is that the spin-spin interaction transfers part of the proton spin to the core neutron spin, i.e. $\left(\left\langle s_{z_{n}}\right\rangle-\left\langle s_{z_{n}}\right\rangle^{o}\right)=-\left(\left\langle s_{z_{p}}\right\rangle-\left\langle s_{z_{p}}\right\rangle^{o}\right)$ and $\left\langle l_{z_{p}}\right\rangle=\left\langle l_{z_{p}}\right\rangle^{o}$. Then we can solve the equation for the magnetic moment and obtain the deviation from the valence model value

$$
\delta \mu-\delta \mu^{o}=-0.11\left(\mu-\mu^{o}\right) \frac{\delta m_{q}}{m_{q}}
$$

To test the stability of the result, we can try different "extreme" assumptions. For example, if the angular momentum exchange occurs exclusively between the proton spin and proton orbital angular momentum, then $\left\langle l_{z_{p}}\right\rangle-\left\langle l_{z_{p}}\right\rangle^{o}=-\left(\left\langle s_{z_{p}}\right\rangle-\left\langle s_{z_{p}}\right\rangle^{o}\right),\left\langle s_{z_{n}}\right\rangle=\left\langle s_{z_{n}}\right\rangle^{o}$. In this case

$$
\delta \mu-\delta \mu^{o}=-0.12\left(\mu-\mu^{o}\right) \frac{\delta m_{q}}{m_{q}}
$$

Finally, we can try an unreasonable assumption that the exchange happens between the proton spin and neutron orbital angular momentum: $\left\langle l_{z_{n}}\right\rangle-\left\langle l_{z_{n}}\right\rangle^{o}=-\left(\left\langle s_{z_{p}}\right\rangle-\right.$ $\left.\left\langle s_{z_{p}}\right\rangle^{o}\right),\left\langle s_{z_{n}}\right\rangle=\left\langle s_{z_{n}}\right\rangle^{o},\left\langle l_{z_{p}}\right\rangle=\left\langle l_{z_{p}}\right\rangle^{o}$. Then

$$
\delta \mu-\delta \mu^{o}=-0.10\left(\mu-\mu^{o}\right) \frac{\delta m_{q}}{m_{q}}
$$


We see that the results are very stable, the difference in the correction to the valence model is about $10 \%$ only. The results for a valence neutron are similar. The coefficients are -0.11 for $\left(\left\langle s_{z_{n}}\right\rangle-\left\langle s_{z_{n}}\right\rangle^{o}\right)=-\left(\left\langle s_{z_{p}}\right\rangle-\right.$ $\left.\left\langle s_{z_{p}}\right\rangle^{o}\right),-0.12$ for $\left\langle l_{z_{n}}\right\rangle-\left\langle l_{z_{n}}\right\rangle^{o}=-\left(\left\langle s_{z_{n}}\right\rangle-\left\langle s_{z_{n}}\right\rangle^{o}\right)$, and -0.09 for $\left\langle l_{z_{p}}\right\rangle-\left\langle l_{z_{p}}\right\rangle^{o}=-\left(\left\langle s_{z_{n}}\right\rangle-\left\langle s_{z_{n}}\right\rangle^{o}\right)$.

To present the final results, we will use a physical approximation which gives results somewhere in between the "extreme" assumptions. The nuclear magnetic moments are reproduced with a reasonable accuracy by the RPA calculations. In the RPA approximation there are two separate conservation laws for the total proton $j_{p}$ and total neutron $j_{n}$ angular momenta (see e.g. [19]). We also assume that total orbital angular momentum $\left\langle l_{z_{n}}\right\rangle+\left\langle l_{z_{p}}\right\rangle$ and total spin $\left\langle s_{z_{n}}\right\rangle+\left\langle s_{z_{p}}\right\rangle$ are conserved (this assumption corresponds to neglect of the spin-orbit interaction). We repeat again that we only need these approximations to obtain specific numbers which are in between "extreme" model values. Then we can write

$$
\begin{aligned}
\left\langle s_{z}\right\rangle^{o} & =\left\langle s_{z_{p}}\right\rangle+\left\langle s_{z_{n}}\right\rangle \\
\left\langle j_{z_{p}}\right\rangle & =\left\langle l_{z_{p}}\right\rangle+\left\langle s_{z_{p}}\right\rangle
\end{aligned}
$$

where $\left\langle j_{z_{p}}\right\rangle=I$ for a valence proton and $\left\langle j_{z_{p}}\right\rangle=0$ for a valence neutron. Using equations (201) and (19) to eliminate $\left\langle l_{z_{p}}\right\rangle$ and $\left\langle s_{z_{p}}\right\rangle$ in equation (14) we get

$$
\begin{gathered}
\mu=g_{n}\left\langle s_{z_{n}}\right\rangle+\left(g_{p}-1\right)\left\langle s_{z_{p}}\right\rangle+\left\langle j_{z_{p}}\right\rangle \\
\left\langle s_{z_{n}}\right\rangle=\frac{\mu-\left\langle j_{z_{p}}\right\rangle-\left(g_{p}-1\right)\left\langle s_{z}\right\rangle^{o}}{g_{n}+1-g_{p}} \\
\left\langle s_{z_{p}}\right\rangle=\left\langle s_{z}\right\rangle^{o}-\left\langle s_{z_{n}}\right\rangle
\end{gathered}
$$

We thus have taken into account both the proton and neutron contributions to the nuclear magnetic moment and can more accurately estimate how a variation in quark mass relates to a variation in $\mu$. From equation (14) we see immediately that

$$
\delta \mu=\delta g_{n}\left\langle s_{z_{n}}\right\rangle+\delta g_{p}\left\langle s_{z_{p}}\right\rangle
$$

and thus

$$
\frac{\delta \mu}{\mu}=\frac{\delta g_{n}}{g_{n}} K_{n}+\frac{\delta g_{p}}{g_{p}} K_{p}
$$

where

$$
K_{n}=\left\{\frac{g_{n}\left\langle s_{z_{n}}\right\rangle}{\mu}\right\} \quad \text { and } \quad K_{p}=\left\{\frac{g_{p}\left\langle s_{z_{p}}\right\rangle}{\mu}\right\}
$$

From the definition of the $g$-factor for free protons and free neutrons, we know $\delta g_{n} / g_{n}=\delta \mu_{n} / \mu_{n}$ and $\delta g_{p} / g_{p}=$ $\delta \mu_{p} / \mu_{p}$. We can now use equations (6) - 9) to explicitly relate the variation in $\mu$ to the variation in quark masses. Thus

$$
\frac{\delta \mu}{\mu}=\kappa_{q} \frac{\delta m_{q}}{m_{q}}+\kappa_{s} \frac{\delta m_{s}}{m_{s}}
$$

where clearly

$$
\begin{gathered}
\kappa_{q}=-0.118 K_{n}-0.087 K_{p} \\
\kappa_{s}=0.0013 K_{n}-0.013 K_{p}
\end{gathered}
$$

We are now in a position to evaluate the coefficients in specific cases. For ${ }^{133} \mathrm{Cs}, I^{\pi}=7 / 2^{+}$and $\mu=2.5820$ and it has a valence proton. Thus $\left\langle j_{z_{p}}\right\rangle=\frac{7}{2}=l-\frac{1}{2}$ and $\left\langle s_{z}\right\rangle^{\circ}=-\frac{7}{18}$. Therefore equations (22) and (23) immediately give us

$$
\begin{aligned}
& \left\langle s_{z_{n}}\right\rangle=-0.103 \\
& \left\langle s_{z_{p}}\right\rangle=-0.286
\end{aligned}
$$

and thus

$$
\frac{\delta \mu}{\mu}=0.152 \frac{\delta g_{n}}{g_{n}}-0.619 \frac{\delta g_{p}}{g_{p}}
$$

giving

$$
\frac{\delta \mu}{\mu}=0.0358 \frac{\delta m_{q}}{m_{q}}+0.00824 \frac{\delta m_{s}}{m_{s}} .
$$

The dependence on the strange quark mass is relatively weak and it is convenient to assume that the relative variation of the strange quark mass is the same as the relative variation in the light quark masses (this assumption is motivated by the Higgs mechanism of mass generation). We restore the explicit notation for the strong-coupling constant and conclude

$$
\frac{\delta \mu}{\mu}=0.0441 \frac{\delta\left(m_{q} / \Lambda_{Q C D}\right)}{\left(m_{q} / \Lambda_{Q C D}\right)} \quad \text { for } \quad{ }^{133} \mathrm{Cs} .
$$

Values for ${ }^{87} \mathrm{Rb},{ }^{199} \mathrm{Hg},{ }^{171} \mathrm{Yb},{ }^{111} \mathrm{Cd}$ and ${ }^{129} \mathrm{Xe}$ can be similarly calculated and are presented in Table

For ${ }^{2} \mathrm{H}$ and ${ }^{3} \mathrm{He}$, the magnetic moments are pretty close to naive values, therefore we have not tried to improve the results.

These results can be summarized in Table II A comparison with the earlier results (using the valence model method) for the total variational relation $\left(\kappa_{q}+\kappa_{s}\right)$ is shown later in Table III] We see that $\delta \mu / \mu$ in the nuclei with a valence proton is very sensitive to the core polarization effects. However, there is no such sensitivity in the nuclei with a valence neutron. To explain this conclusion one should note that a neutron does not give an orbital contribution to the nuclear magnetic moment. The orbital contribution of core protons is relatively small. As a result, the core polarization effect changes $\delta \mu$ and $\mu$ in a similar way, i.e. it practically does not change their ratio. In the nuclei with a valence neutron

$$
\frac{\delta \mu}{\mu}=-0.117 \frac{\left\langle s_{z_{n}}\right\rangle-1.25\left\langle s_{z_{p}}\right\rangle}{\left\langle s_{z_{n}}\right\rangle-1.20\left\langle s_{z_{p}}\right\rangle} \frac{\delta m_{q}}{m_{q}}
$$


TABLE II: Variation in $\mu$ incorporating the effect of non-valence nucleons in various atoms. $\left\langle s_{z}\right\rangle^{\circ}$ is the spin expectation value, $\left\langle s_{z_{n}}\right\rangle$ and $\left\langle s_{z_{p}}\right\rangle$ are (either, depending on nucleus) the valence and non-valance nucleon contributions to this spin, $K_{n}$ and $K_{p}$ are defined in equation 25 and $\kappa_{q}$ and $\kappa_{s}$ are defined in equation 26]

\begin{tabular}{c|ccccccccc}
\hline \hline Atom & ${ }^{1} \mathrm{H}$ & ${ }^{2} \mathrm{H}$ & ${ }^{3} \mathrm{He}$ & ${ }^{87} \mathrm{Rb}$ & ${ }^{111} \mathrm{Cd}$ & ${ }^{129} \mathrm{Xe}$ & ${ }^{133} \mathrm{Cs}$ & ${ }^{171} \mathrm{Yb}$ & ${ }^{199} \mathrm{Hg}$ \\
\hline$\left\langle s_{z}\right\rangle^{o}$ & 0 & 1 & 0.5 & 0.5 & 0.5 & 0.5 & -0.389 & -0.167 & -0.167 \\
\hline$\left\langle s_{z_{n}}\right\rangle$ & 0 & 0.5 & 0.5 & 0.124 & 0.343 & 0.365 & -0.103 & -0.150 & -0.151 \\
\hline$\left\langle s_{z_{p}}\right\rangle$ & 0.5 & 0.5 & 0 & 0.376 & 0.157 & 0.135 & -0.286 & -0.017 & -0.016 \\
\hline$K_{n}$ & - & - & - & -0.172 & 2.21 & 1.80 & 0.152 & 1.16 & 1.14 \\
\hline$K_{p}$ & - & - & - & 0.764 & -1.47 & -0.969 & -0.619 & -0.194 & -0.173 \\
\hline$\kappa_{q}$ & -0.087 & -0.020 & -0.118 & -0.046 & -0.133 & -0.128 & 0.036 & -0.120 & -0.120 \\
\hline$\kappa_{s}$ & -0.013 & -0.044 & 0.0013 & -0.010 & 0.022 & 0.015 & 0.008 & 0.004 & 0.004 \\
\hline$\kappa_{q}+\kappa_{s}$ & -0.100 & -0.064 & -0.117 & -0.056 & -0.111 & -0.113 & 0.044 & -0.116 & -0.116 \\
\hline
\end{tabular}

\section{Effect of variation of the the spin-spin interaction}

In previous subsection we have not taken into account that $\left\langle s_{z_{p}}\right\rangle$ and $\left\langle s_{z_{n}}\right\rangle$ may depend on the quark mass. However, this dependence appears since the spin-spin interaction depends on the quark masses. Below we want to perform a rough estimate of this effect using the oneboson-exchange model of the strong interaction.

Consider, for example, a nucleus with a valence proton. As above we assume that the total spin of nucleons is conserved and so

$$
\left\langle s_{z}\right\rangle^{o}=\left\langle s_{z_{p}}\right\rangle+\left\langle s_{z_{n}}\right\rangle
$$

where $\left\langle s_{z}\right\rangle^{o}$ is again the valence model value. It is convenient to use the following notations:

$$
\begin{aligned}
\left\langle s_{z_{n}}\right\rangle & =b\left\langle s_{z}\right\rangle^{o} \\
\left\langle s_{z_{p}}\right\rangle & =(1-b)\left\langle s_{z}\right\rangle^{o}
\end{aligned}
$$

where $\mathrm{b}$ is a coefficient determined by the spin-spin interaction. We need to determine the dependence of $b$ on $m_{q} / \Lambda_{Q C D}$ to complete our calculations. It can be estimated using perturbation theory as

$$
b \sim\left(\frac{\left\langle o\left|V_{s s}\right| k\right\rangle}{E_{o}-E_{k}}\right)^{2}
$$

where

$$
V_{s s}=V\left(\left|\mathbf{r}_{1}-\mathbf{r}_{2}\right|\right) \mathbf{S}_{1} \cdot \mathbf{S}_{2}
$$

and $E_{o}-E_{k}$ is the spin-orbit splitting (see e.g. [19]). As $m_{q} \rightarrow 0, E_{o}-E_{k}$ remains finite and so it can only have a weak dependence on $m_{q} / \Lambda_{Q C D}$. The major dependence comes from the $\pi$-meson mass which vanishes in the chiral limit $m_{q} \rightarrow 0$. According to review [20] the $\pi$-meson exchange gives about $1 / 3$ of the spin-spin interaction. The other most significant contribution is given by the $\rho$-meson exchange. We neglect other meson contributions and assume that the remaining $2 / 3$ of the spin-spin interaction is given by the $\rho$-meson. The result is not very sensitive to this assumption since the $\rho$-meson and other vector mesons have approximately the same and rather weak sensitivity to a variation in $m_{q}$. According to Ref. 21]

$$
\frac{\delta m_{\rho}}{m_{\rho}}=0.021 \frac{\delta m_{q}}{m_{q}}
$$

whereas for the $\pi-$ meson

$$
m_{\pi} \sim \sqrt{m_{q} \Lambda_{Q C D}}
$$

so we have

$$
\frac{\delta m_{\pi}}{m_{\pi}}=\frac{1}{2} \frac{\delta m_{q}}{m_{q}}
$$

The dominating contribution is therefore given by $\pi$ meson exchange. The exchange contribution of $\pi^{ \pm}$is small due to the small overlap between $\psi_{p}(r)$ and $\psi_{n}(r)$ and is not important. The main contribution is when a neutron is excited through $\pi^{0}$ exchange into a spin-orbit doublet, $j=l+\frac{1}{2}$ to $j=l-\frac{1}{2}$. The strongest dependence thus originates from the $\pi^{0}$ pion mass.

The momentum space representation of the nucleonnucleon interaction due to a $\pi$-meson is

$$
V_{\pi}(q)=g_{\pi}^{2}\left(\vec{\tau}_{1} \cdot \vec{\tau}_{2}\right)\left(\vec{\sigma}_{1} \cdot \mathbf{q}\right)\left(\vec{\sigma}_{2} \cdot \mathbf{q}\right) \frac{1}{m_{\pi}^{2}+q^{2}}
$$

where $\mathbf{q}$ is the momentum transfer $\mathbf{q}=\mathbf{p}_{1}-\mathbf{p}_{2}, \vec{\tau}$ is the isotopic spin and $\vec{\sigma}$ is the Pauli spin matrix.

We separate this into tensor and scalar parts

$$
\begin{gathered}
\left(\vec{\sigma}_{1} \cdot \mathbf{q}\right)\left(\vec{\sigma}_{2} \cdot \mathbf{q}\right)=\left\{\left(\vec{\sigma}_{1} \cdot \mathbf{q}\right)\left(\vec{\sigma}_{2} \cdot \mathbf{q}\right)-\frac{1}{3} \vec{\sigma}_{1} \cdot \vec{\sigma}_{2} q^{2}\right\} \\
+\left\{\frac{1}{3} \vec{\sigma}_{1} \cdot \vec{\sigma}_{2} q^{2}\right\}
\end{gathered}
$$


The scalar part of the interaction we are interested in becomes

$$
\begin{array}{r}
V_{\pi}^{\text {scalar }}(q)=\frac{g_{\pi}^{2}}{3}\left(\vec{\tau}_{1} \cdot \vec{\tau}_{2}\right)\left(\vec{\sigma}_{1} \cdot \vec{\sigma}_{2}\right) \frac{q^{2}}{m_{\pi}^{2}+q^{2}} \\
=\frac{g_{\pi}^{2}}{3}\left(\vec{\tau}_{1} \cdot \vec{\tau}_{2}\right)\left(\vec{\sigma}_{1} \cdot \vec{\sigma}_{2}\right)\left(1-\frac{m_{\pi}^{2}}{m_{\pi}^{2}+q^{2}}\right)
\end{array}
$$

Fourier transformation of this and letting $r_{12}=\left|\mathbf{r}_{2}-\mathbf{r}_{1}\right|$ will give us the coordinate space representation

$$
\begin{aligned}
V_{\pi}^{\text {scalar }}\left(r_{12}\right)= & \frac{g_{\pi}^{2}}{3}\left(\vec{\tau}_{1} \cdot \vec{\tau}_{2}\right)\left(\vec{\sigma}_{1} \cdot \vec{\sigma}_{2}\right) \times \\
& {\left[-4 \pi \delta\left(r_{12}\right)+m_{\pi}^{2} \frac{1}{r_{12}} e^{-m_{\pi} r_{12}}\right] }
\end{aligned}
$$

As $m_{q} \rightarrow 0, g_{\pi}$ is finite and so we can neglect its dependence on $m_{q} / \Lambda_{Q C D}$. Now the strong force short range repulsion (implying proton and neutron hard cores) needs to be taken into account. It means that $r_{12} \neq 0$ and hence $\delta\left(r_{12}\right)=0$. Nucleon core repulsion is incorporated into the interaction using the factor $f\left(r_{12}\right)$ which is presented e.g. in Ref. 222]

$$
\tilde{V}\left(r_{12}\right)=\left[f\left(r_{12}\right)\right]^{2} V\left(r_{12}\right)
$$

where

$$
f\left(r_{12}\right)=1-e^{-1.1 \rho^{2}}\left(1-0.68 \rho^{2}\right) \quad, \rho=\frac{r_{12}}{\mathrm{fm}} .
$$

Clearly, this factor restricts nucleon interaction at very short ranges, with $f\left(r_{12}=0\right)=0$, yet its effect is minimal at larger distances since $f\left(r_{12}\right) \approx 1$ for $r_{12}>1 \mathrm{fm}$. It is this factor which results in a non-zero dependence of $b$ on $m_{q} / \Lambda_{Q C D}$.

Therefore we have the effective spin-spin interaction

$$
V_{s s}=V_{o}\left(r_{12}\right)\left(\mathbf{S}_{1} \cdot \mathbf{S}_{2}\right) \times \text { constant }
$$

where

$$
V_{o}\left(r_{12}\right)=m_{\pi}^{2} e^{-m_{\pi} r_{12}} \frac{\left[f\left(r_{12}\right)\right]^{2}}{r_{12}}
$$

We can obtain the short range limit of this effective interaction (which takes into account the finite size of the nucleons, that is the short-range repulsion). For clarity in our equations, we define $B, S$ and $S_{1}$. First let

$$
V_{o}\left(r_{12}\right) \approx B \delta\left(r_{12}\right)
$$

so that we have

$$
B=B \int \delta\left(r_{12}\right) d^{3} r_{12}=\int V_{o}\left(r_{12}\right) d^{3} r_{12}
$$

Also let

$$
\begin{aligned}
S & =\frac{1}{m_{\pi}^{2}} \int V_{o}\left(r_{12}\right) d^{3} r_{12} \\
& =4 \pi \int_{0}^{\infty} e^{-m_{\pi} r_{12}}\left[f\left(r_{12}\right)\right]^{2} r_{12} d r_{12}
\end{aligned}
$$

and

$$
S_{1}=4 \pi m_{\pi} \int_{0}^{\infty} e^{-m_{\pi} r_{12}}\left[f\left(r_{12}\right)\right]^{2}\left[r_{12}\right]^{2} d r_{12}
$$

so that

$$
\frac{\partial S}{\partial m_{\pi}}=-\frac{S_{1}}{m_{\pi}} .
$$

Thus

$$
\frac{\delta S}{S}=-\frac{\delta m_{\pi}}{m_{\pi}} \frac{S_{1}}{S}
$$

From these definitions, we have

$$
B=m_{\pi}^{2} \cdot S
$$

and so

$$
\frac{\delta B}{B}=2 \frac{\delta m_{\pi}}{m_{\pi}}+\frac{\delta S}{S}=2 \frac{\delta m_{\pi}}{m_{\pi}}-\frac{\delta m_{\pi}}{m_{\pi}} \frac{S_{1}}{S}
$$

Recalling that the $\pi$-meson contributes only $1 / 3$ to the spin-spin interaction, we have:

$$
b \sim\left(\frac{\left\langle o\left|V_{s s}\right| k\right\rangle}{E_{o}-E_{k}}\right)^{2} \sim \frac{1}{3} B^{2} \quad \text { and with } \quad \frac{\delta m_{\pi}}{m_{\pi}}=\frac{1}{2} \frac{\delta m_{q}}{m_{q}}
$$

we see

$$
\frac{\delta b}{b}=\frac{2}{3}\left(2-\frac{S_{1}}{S}\right) \frac{\delta m_{\pi}}{m_{\pi}}=\frac{1}{3}\left(2-\frac{S_{1}}{S}\right) \frac{\delta m_{q}}{m_{q}}
$$

The integrals for $S$ and $S_{1}$ can be evaluated using $m_{\pi}=$ $m_{\pi^{o}}=135 \mathrm{MeV}=0.68 \mathrm{fm}^{-1}$ to give

$$
\frac{S_{1}}{S}=2.17 \text {. }
$$

This gives a small number for $\frac{S_{1}}{S}-2=0.17$, therefore the result may seem to be unstable. It is useful to clarify this point using a simpler analytical model for the repulsive core with $f^{2}=1-\exp (-k r)$ which gives

$$
\begin{gathered}
\frac{S_{1}}{S}=2 \frac{1-R^{3}}{1-R^{2}} \\
R=\frac{m_{\pi}}{k+m_{\pi}} .
\end{gathered}
$$

Any value of $k>m_{\pi}$ gives $R<0.5$ and so gives a small difference for $\frac{S_{1}}{S}-2$. Therefore, the small value of this difference does not indicate any strong instability. If we take $k=1.1 \mathrm{fm}^{-1}$ (the same value of the core radius which we used in the more sophisticated model for $f$ described above), we obtain $\frac{S_{1}}{S}=2.2$, i.e. practically the same result as above. Thus, the result does not have any strong model dependence.

Using $\frac{S_{1}}{S}=2.17$ we obtain the following $\pi$-meson contribution to the variation of $b$ :

$$
\left(\frac{\delta b}{b}\right)_{\pi}=-0.057 \frac{\delta m_{q}}{m_{q}}
$$


Similarly, for the $\rho$-meson we obtained $S 1 / S=3.77$ and

$$
\left(\frac{\delta b}{b}\right)_{\rho}=-2.4 \frac{\delta m_{\rho}}{m_{\rho}}=-0.05 \frac{\delta m_{q}}{m_{q}} .
$$

The final estimate is

$$
\left(\frac{\delta b}{b}\right)_{\text {total }}=-0.11 \frac{\delta m_{q}}{m_{q}} .
$$

Note that if we assume that the spin-spin interaction is completely dominated by the $\pi$-meson exchange the result (-0.17) would not be very different.

Returning to the magnetic moment, we have for the case of a valence proton:

$$
\begin{gathered}
\mu=g_{n} b\left\langle s_{z}\right\rangle^{o}+\left(g_{p}-1\right)(1-b)\left\langle s_{z}\right\rangle^{o}+\left\langle j_{z_{p}}\right\rangle \\
\frac{\delta \mu}{\mu}=\frac{\delta g_{n}}{g_{n}} K_{n}+\frac{\delta g_{p}}{g_{p}} K_{p}+\frac{\delta b}{b} K_{b_{p}} . \\
K_{b_{p}}=\frac{\left(g_{n}-g_{p}+1\right)\left\langle s_{z_{n}}\right\rangle}{\mu}
\end{gathered}
$$

Similarly, for the case of a valence neutron:

$$
\begin{gathered}
\mu=g_{n}(1-b)\left\langle s_{z}\right\rangle^{o}+\left(g_{p}-1\right) b\left\langle s_{z}\right\rangle^{o} . \\
\frac{\delta \mu}{\mu}=\frac{\delta g_{n}}{g_{n}} K_{n}+\frac{\delta g_{p}}{g_{p}} K_{p}+\frac{\delta b}{b} K_{b_{n}} . \\
K_{b_{n}}=\frac{\left(g_{p}-g_{n}-1\right)\left\langle s_{z_{p}}\right\rangle}{\mu}
\end{gathered}
$$

The dependence of $\delta \mu / \mu$ on the spin-spin interaction (via the $K_{b}$ term) can now be seen to be quite significant. It depends on three values. The first is the common factor $\left(g_{p}-g_{n}-1\right)=8.41$, which is large. Next, it depends on the value of the effective spin of the non-valence nucleons, which indicates the extent of the spin-spin interaction. It is most significant when the experimental value for $\mu$ deviates greatly from the valence model value. Third, it depends on the nuclear magnetic moment and so is further enhanced when dealing with nuclei with small magnetic moments (e.g. ${ }^{111} \mathrm{Cd}$ has $\mu=-0.5949$ whereas ${ }^{133} \mathrm{Cs}$ has $\left.\mu=2.582\right)$.

We now have a modified version of equation (26) which includes the term $-0.11 K_{b}$ to account for the variation of the spin-spin interaction itself:

$$
\begin{array}{r}
\frac{\delta \mu}{\mu}=\kappa \frac{\delta\left(m_{q} / \Lambda_{Q C D}\right)}{\left(m_{q} / \Lambda_{Q C D}\right)} \\
\kappa=-0.12 K_{n}-0.10 K_{p}-0.11 K_{b}
\end{array}
$$

For ${ }^{133} \mathrm{Cs}$, we use the values obtained earlier to get

$$
K_{b_{p}}=\frac{\left(g_{n}-g_{p}+1\right)\left\langle s_{z_{n}}\right\rangle}{\mu}=0.335
$$

giving

$$
\frac{\delta \mu}{\mu}=0.009 \frac{\delta\left(m_{q} / \Lambda_{Q C D}\right)}{\left(m_{q} / \Lambda_{Q C D}\right)}
$$

All calculations for ${ }^{139} \mathrm{La},{ }^{87} \mathrm{Rb},{ }^{199} \mathrm{Hg},{ }^{171} \mathrm{Yb},{ }^{111} \mathrm{Cd}$ and ${ }^{129} \mathrm{Xe}$ are similar to the method used for ${ }^{133} \mathrm{Cs}$. The results are presented in Table III which summarizes the three methods used. Method A was the first method discussed and used the theoretical nuclear magnetic moment of just the valence nucleon. Method B included the contribution from non-valence nucleons to the nuclear magnetic moment. Method $\mathrm{C}$ further included the effect of a variation in quark mass on the spin-spin interaction itself. It shows the significance of the spin-spin interaction on how $\mu$ varies with quark masses, with sign reversal for some nuclei.

\section{Limits on the variation of the fine structure constant $\alpha$ and $\left(m_{q} / \Lambda_{Q C D}\right)$ using recent atomic clock experiments}

We can now estimate the time dependence of the ratio of the hyperfine transition frequencies to variations in $m_{q} / \Lambda_{Q C D}$. The results for each atom $\mathrm{M}$ can be presented using the parameter $V$ as defined earlier with equation (11), with the values of $K_{r e l}$ and $\kappa$ for the atoms considered here summarized in Table IV]

$$
\frac{\delta V(M)}{V(M)}=\frac{\delta(A / E)}{A / E}=\alpha^{2+K_{\text {relM }}}\left(\frac{m_{q}}{\Lambda_{Q C D}}\right)^{\kappa_{M}} \frac{m_{e}}{m_{p}} .
$$

For two atoms, $M_{1}$ and $M_{2}$, the dependence of the ratio of the frequencies $A\left(M_{1}\right) / A\left(M_{2}\right)$ can be presented as the ratio $X$

$$
\begin{aligned}
X\left(M_{1} / M_{2}\right) & =\frac{V\left(M_{1}\right)}{V\left(M_{2}\right)} \\
& =\alpha^{K_{\text {rel } M_{1}}-K_{\text {rel } M_{2}}}\left(\frac{m_{q}}{\Lambda_{Q C D}}\right)^{\kappa_{M_{1}}-\kappa_{M_{2}}}(48)
\end{aligned}
$$

For $A\left({ }^{87} \mathrm{Rb}\right) / A\left({ }^{133} \mathrm{Cs}\right)$, we have

$$
X(\mathrm{Rb} / \mathrm{Cs})=\alpha^{-0.49}\left(\frac{m_{q}}{\Lambda_{Q C D}}\right)^{-0.025}
$$

and the result of measurements by [8] can be presented as a limit on the variation of $\mathrm{X}$ :

$$
\frac{1}{X(\mathrm{Rb} / \mathrm{Cs})} \frac{d X(\mathrm{Rb} / \mathrm{Cs})}{d t}=(-0.5 \pm 5.3) \times 10^{-16} / \mathrm{yr} .
$$

For $A\left({ }^{133} \mathrm{Cs}\right) / A\left({ }^{1} \mathrm{H}\right)$, we have

$$
X(\mathrm{Cs} / \mathrm{H})=\alpha^{0.83}\left(\frac{m_{q}}{\Lambda_{Q C D}}\right)^{0.109}
$$


TABLE III: Comparison of results for $\kappa$ (see equation (47)) for the three methods used in various nuclei

\begin{tabular}{c|rrrrrrr}
\hline \hline Atom & ${ }^{87} \mathrm{Rb}$ & ${ }^{111} \mathrm{Cd}$ & ${ }^{129} \mathrm{Xe}$ & ${ }^{133} \mathrm{Cs}$ & ${ }^{139} \mathrm{La}$ & ${ }^{171} \mathrm{Yb}$ & ${ }^{199} \mathrm{Hg}$ \\
\hline Method A* & -0.074 & -0.117 & -0.117 & 0.127 & 0.127 & -0.117 & -0.117 \\
\hline Method B* & -0.056 & -0.111 & -0.113 & 0.044 & 0.032 & -0.116 & -0.116 \\
\hline Method C & -0.016 & 0.125 & 0.042 & 0.009 & -0.008 & -0.085 & -0.088 \\
\hline
\end{tabular}

* Method A considered only the valence nucleon, Method B includes the non-valence nucleons, Method C further includes the effect of quark mass on the spin-spin interaction.

TABLE IV: Summary of final results showing the relative sensitivity of the hyperfine relativistic factor to a variation in $\alpha$ (parameter $K_{r e l}$ ) and the relative sensitivity of the nuclear magnetic moment to a variation in the quark mass/strong interaction scale $m_{q} / \Lambda_{Q C D}$ (parameter $\kappa$ ). These values can be used in equation (48).

\begin{tabular}{c|cccccccccc}
\hline \hline Atom & ${ }_{1}^{1} \mathrm{H}$ & ${ }_{1}^{2} \mathrm{H}$ & ${ }_{2}^{3} \mathrm{He}$ & ${ }_{37}^{87} \mathrm{Rb}$ & ${ }_{48}^{111} \mathrm{Cd}$ & ${ }_{54}^{129} \mathrm{Xe}$ & ${ }_{55}^{133} \mathrm{Cs}$ & ${ }_{57}^{139} \mathrm{La}$ & ${ }_{70}^{171} \mathrm{Yb}$ & ${ }_{80}^{199} \mathrm{Hg}$ \\
\hline$K_{\text {rel }}$ & 0 & 0 & 0 & 0.34 & 0.6 & 0.8 & 0.83 & 0.9 & 1.5 & 2.28 \\
\hline$\kappa$ & -0.100 & -0.064 & -0.117 & -0.016 & 0.125 & 0.042 & 0.009 & -0.008 & -0.085 & -0.088 \\
\hline
\end{tabular}

and the result of the measurements in Ref. [9] may be presented as

$$
\left|\frac{1}{X(\mathrm{Cs} / \mathrm{H})} \frac{d X(\mathrm{Cs} / \mathrm{H})}{d t}\right|<5.5 \times 10^{-14} / y r .
$$

For $A\left({ }^{171} \mathrm{Yb}^{+}\right) / A\left({ }^{133} \mathrm{Cs}\right)$, we have

$$
X\left(\mathrm{Yb}^{+} / \mathrm{Cs}\right)=\alpha^{0.67}\left(\frac{m_{q}}{\Lambda_{Q C D}}\right)^{-0.093}
$$

and the result of measurements by [10] can be presented as a limit on the variation of $\mathrm{X}$ :

$$
\frac{1}{X\left(\mathrm{Yb}^{+} / \mathrm{Cs}\right)} \frac{d X\left(\mathrm{Yb}^{+} / \mathrm{Cs}\right)}{d t}=(2.8 \pm 2.9) \times 10^{-14} / \mathrm{yr} .
$$

For $A\left({ }^{199} \mathrm{Hg}\right) / A\left({ }^{1} \mathrm{H}\right)$, we have

$$
X(\mathrm{Hg} / \mathrm{H})=\alpha^{2.28}\left(\frac{m_{q}}{\Lambda_{Q C D}}\right)^{0.012}
$$

and the result of measurements by [1] can be presented as a limit on the variation of $\mathrm{X}$ :

$$
\left|\frac{1}{X(\mathrm{Hg} / \mathrm{H})} \frac{d X(\mathrm{Hg} / \mathrm{H})}{d t}\right|<8 \times 10^{-14} / \mathrm{yr} .
$$

The optical clock transition energy $\mathrm{E}(\mathrm{Hg})(\lambda=282 \mathrm{~nm})$ in the $\mathrm{Hg}^{+}$ion can be presented in the form:

$$
E(\mathrm{Hg})=\text { const } \times\left(\frac{m_{e} e^{4}}{\hbar^{2}}\right) F_{r e l}(Z \alpha)
$$

and calculations by Ref [16] gives

$$
\frac{\delta E(\mathrm{Hg})}{E(\mathrm{Hg})}=-3.2 \frac{\delta \alpha}{\alpha}
$$

corresponding to $V(\mathrm{Hg} \mathrm{Opt})=\alpha^{-3.2}$. Variation of the ratio of the hyperfine splitting $A(\mathrm{Cs})$ is given by

$$
V(\mathrm{Cs})=\alpha^{2.83}\left(\frac{m_{q}}{\Lambda_{Q C D}}\right)^{0.009}\left(\frac{m_{e}}{m_{p}}\right) .
$$

The relative variation of the electron to proton mass ratio can be described by [18]

$$
X\left(m_{e} / m_{p}\right)=\left(\frac{m_{q}}{\Lambda_{Q C D}}\right)^{-0.037}\left(\frac{m_{s}}{\Lambda_{Q C D}}\right)^{-0.011} \frac{m_{e}}{\Lambda_{Q C D}} .
$$

giving

$$
V(\mathrm{Cs})=\alpha^{2.83}\left(\frac{m_{q}}{\Lambda_{Q C D}}\right)^{-0.039}\left(\frac{m_{e}}{\Lambda_{Q C D}}\right)
$$

Variation of the ratio of the hyperfine splitting $A(\mathrm{Cs})$ to this optical transition energy is given by:

$$
\begin{array}{r}
X(\mathrm{Opt})=\frac{V(\mathrm{Cs})}{V(\text { Hg Opt })}=\alpha^{6}\left(\frac{m_{q}}{\Lambda_{Q C D}}\right)^{0.009}\left(\frac{m_{e}}{m_{p}}\right) \\
=\alpha^{6}\left(\frac{m_{q}}{\Lambda_{Q C D}}\right)^{-0.039}\left(\frac{m_{e}}{\Lambda_{Q C D}}\right)
\end{array}
$$

and the result of measurements by [12] can be presented as a limit on the variation of $\mathrm{X}$ :

$$
\left|\frac{1}{X(\mathrm{Opt})} \frac{d X(\mathrm{Opt})}{d t}\right|<7 \times 10^{-15} / \mathrm{yr} \text {. }
$$

For the $1 s-2 s$ transition in hydrogen the relativistic corrections are negligible, i.e. $V(\mathrm{H} \mathrm{Opt})=\alpha^{0}$. Variation of the ratio of the hyperfine splitting $A(\mathrm{Cs})$ to this optical transition energy is given by:

$$
\begin{array}{r}
X(\mathrm{Opt})=\frac{V(\mathrm{Cs})}{V(\mathrm{H} \mathrm{Opt})}=\alpha^{2.83}\left(\frac{m_{q}}{\Lambda_{Q C D}}\right)^{0.009} \frac{m_{e}}{m_{p}} \\
=\alpha^{2.83}\left(\frac{m_{q}}{\Lambda_{Q C D}}\right)^{-0.039}\left(\frac{m_{e}}{\Lambda_{Q C D}}\right)
\end{array}
$$


and the result of measurements by 13 can be presented as a limit on the variation of $\mathrm{X}$ :

$$
\frac{1}{X(\mathrm{Cs} / \mathrm{H} \mathrm{Opt})} \frac{d X(\mathrm{Cs} / \mathrm{H} \mathrm{Opt})}{d t}=(3.2 \pm 6.3) \times 10^{-15} / \mathrm{yr} \text {. }
$$

For the optical clock transition energy $\mathrm{E}(\mathrm{Yb})(\lambda=$ $436 \mathrm{~nm})$ in the $\mathrm{Yb}^{+}$ion calculations by Ref 23] gives

$$
\frac{\delta E(\mathrm{Yb})}{E(\mathrm{Yb})}=0.88 \frac{\delta \alpha}{\alpha}
$$

corresponding to $V(\mathrm{Yb} O \mathrm{Opt})=\alpha^{0.88}$. Variation of the ratio of the hyperfine splitting $A(\mathrm{Cs})$ to this optical transition energy is given by:

$$
\begin{array}{r}
X(\mathrm{Opt})=\frac{V(\mathrm{Cs})}{V(\mathrm{Yb} \mathrm{Opt})}=\alpha^{1.95}\left(\frac{m_{q}}{\Lambda_{Q C D}}\right)^{0.009} \frac{m_{e}}{m_{p}} \\
=\alpha^{1.95}\left(\frac{m_{q}}{\Lambda_{Q C D}}\right)^{-0.039}\left(\frac{m_{e}}{\Lambda_{Q C D}}\right)
\end{array}
$$

and the result of measurements by [14] can be presented as a limit on the variation of $\mathrm{X}$ :

$$
\frac{1}{X(\mathrm{Opt})} \frac{d X(\mathrm{Opt})}{d t}=(1.2 \pm 4.4) \times 10^{-15} / \mathrm{yr}
$$

Other combinations have been suggested as possible areas of research. For $A\left({ }^{129} \mathrm{Xe}\right) / A\left({ }^{3} \mathrm{He}\right)$, we have

$$
X(\mathrm{Xe} / \mathrm{He})=\alpha^{0.8}\left(\frac{m_{q}}{\Lambda_{Q C D}}\right)^{0.159}
$$

while for $A\left({ }^{1} \mathrm{H}\right) / A\left({ }^{2} \mathrm{H}\right)$, we have

$$
X\left({ }^{1} \mathrm{H} /{ }^{2} \mathrm{H}\right)=\left(\frac{m_{q}}{\Lambda_{Q C D}}\right)^{-0.036}
$$

One can use Table IV to predict which hyperfine transitions will be most sensitive to a variation in $\alpha$. The greatest effect will be seen for ratios between atoms with the greatest difference in values of $K_{r e l}$ and $\kappa$, especially if relation (II) were correct. Clearly, it would be best to test ratios of elements with opposite signs for $\kappa$ so that the effects are more pronounced.

The effect of the spin-spin interaction is to reduce the sensitivity of Cs to a variation in quark mass and enhance the sensitivity of other nuclei such as Cd. Because the spin-spin interaction is so strong for $\mathrm{Cd}$, with its magnetic moment of -0.59 being quite different to the valence model value of -1.9 , it may be quite sensitive to a variation in quark mass. In Ref. 6], the importance of Cd was motivated by its small magnetic moment. This could enhance its sensitivity to a variation of the fundamental constants. We have not actually obtained any enhancement, and the absolute value of $|\kappa|$ in $\mathrm{Cd}$ is comparable to the valence model value. However, due to the spin-spin interaction it has an opposite sign relative to some other nuclei with large $|\kappa|$. For example, consider
$A(\mathrm{Cd}) / A(\mathrm{H})$ and $A(\mathrm{Cd}) / A(\mathrm{He})$, with each ratio involving opposite signs for $\kappa$ :

$$
X(\mathrm{Cd} / \mathrm{H})=\alpha^{0.6}\left(\frac{m_{q}}{\Lambda_{Q C D}}\right)^{0.23}
$$

$$
X(\mathrm{Cd} / \mathrm{He})=\alpha^{0.6}\left(\frac{m_{q}}{\Lambda_{Q C D}}\right)^{0.24}
$$

Note that if relation (11) were correct, the variation of $X$ may be dominated by $m_{q} / \Lambda_{Q C D}$ : for $A(\mathrm{Cd}) / A(\mathrm{H})$, $X(\mathrm{Cd} / \mathrm{H}) \propto \alpha^{9}$.

\section{CONCLUSIONS}

The results of this work are presented in the previous section. Table IV provides one with the numbers needed for the interpretation of the measurements. Below, we would like to formulate a few conclusions which correct some misconceptions in the existing literature and may help to plan future experiments and calculations.

- There is no such thing as a "model-independent interpretation of measurements" if one uses the valence model (Schmidt) values of the nuclear magnetic moments. The valence model cannot even guarantee the order of magnitude and sign of the effect.

The situation may be improved by presenting the nuclear magnetic moment as a linear combination of the neutron, proton and orbital magnetic moments. However, even this method does not guarantee high accuracy since the expansion coefficients in this linear combination depend on the ratio $m_{q} / \Lambda_{Q C D}$. A consistent interpretation of the measurements requires the calculation of the dependence of nuclear magnetic moment on this parameter.

- A small value for the nuclear magnetic moment does not guarantee an enhancement of the sensitivity to $m_{q} / \Lambda_{Q C D}$. However, a large deviation from the valence model value should increase the error in the calculated sensitivity.

- The dependence on $m_{q} / \Lambda_{O C D}$ of the nuclear magnetic moments of ${ }_{37}^{87} \mathrm{Rb},{ }_{55}^{133} \mathrm{Cs},{ }_{57}^{139} \mathrm{La}$ and ${ }_{54}^{129} \mathrm{Xe}$ is strongly suppressed by the many-body corrections - see Table III One cannot guarantee high accuracy of calculation in this situation. However, it is probably not important since the suppression means that the contribution of these magnetic moments to the final effect of the variation is small. The effect will be dominated by the variation of $\alpha, m_{e} / m_{p}$ or another magnetic moment. For ${ }_{48}^{111} \mathrm{Cd}$ (where the magnetic moment is small) the effect is not suppressed but it is of opposite sign to the valence model value. For ${ }_{70}^{171} \mathrm{Yb}$ and ${ }_{80}^{199} \mathrm{Hg}$ the deviations from the valence model are small. Naive calculations give also reasonable results for ${ }_{1}^{2} \mathrm{H}$ and ${ }_{2}^{3} \mathrm{He}$. 


\section{ACKNOWLEDGMENTS}

This work was supported by the Australian Research Council. VVF is grateful to A. Brown, E. Eppelbaum,
S.G. Karshenboim, E. Peik and V.G. Zelevinsky for useful discussions.
[1] J-P. Uzan, Rev. Mod. Phys. 75, 403 (2003);

[2] P. Langacker, G. Segre, and M.J. Strassler Phys. Lett. B 528, 121 (2002). see also X. Calmet and H. Fritzsch, Eur. Phys. J.C 24, 639 (2002); W.J. Marciano, Phys. Rev. Lett. 52, 489 (1984).

[3] V.V. Flambaum and E.V. Shuryak, Phys. Rev. D 65, 103503 (2002).

[4] V.F. Dmitriev and V.V. Flambaum, Phys. Rev. D 67, 063513 (2003).

[5] V.V. Flambaum and E.V. Shuryak, Phys. Rev. D 67, 083507 (2003).

[6] S.G. Karshenboim, Can. J. Phys. 78, 639 (2000).

[7] H. Marion et al., Phys. Rev. Lett. 90, 150801 (2003).

[8] S. Bize et al., arXiv:physics/0502117 1.

[9] N.A. Demidov et al, in Proceedings of the 6th European Frequency and Time Forum. Noordwijk, the Netherlands, 1992 (European Space Agency, Noordwijk,1992), pp.409414. L.A. Breakiron, in Proceedings of the 25th Annual Precise Time Interval Applications and Planning Meeting, NASA conference publication No. 3267 [U.S. Naval Observatory Time Service Department (TSS1), Washington DC, 1993], pp. 401-412.

[10] S.G. Karshenboim, V. Flambaum, E. Peik arXiv:physics/0410074 published in Handbook of Atomic, Molecular and Optical Physics (Ed. G.W.F. Drake, Springer, Berlin 2005), Ch 30, p.459. This result is based on the measurements in P.T.H. Fisk et al. IEEE Trans.UFFC 44, 344 (1997); P.T.H. Fisk, Rep. Prog.
Phys. 60, 761 1997; R.B. Warrington et al. Proceedings of 6th Symposium Frequency Standards and Metrology (World Scientific, Singapore 2002), p. 297.

[11] J.D. Prestage, R.L. Tjoelker and L. Maleki, Phys. Rev. Lett. 74, 3511 (1995).

[12] S. Bize et al., Phys. Rev. Lett. 90, 150802 (2003).

[13] M. Fischer et al. arXiv:physics/0311128

[14] E. Peik, B. Lipphardt, H. Schnatz, T. Schneider, Chr. Tamm, S.G. Karshenboim, arXiv:physics/0504101

[15] V.V. Flambaum, arXiv:physics/0601034

[16] V.A. Dzuba, V.V. Flambaum, and J.K. Webb, Phys. Rev. A 59, 230 (1999); V.A. Dzuba (private communication).

[17] V.V. Flambaum, arXiv:physics/0302015 (2003)

[18] V.V. Flambaum, D.B. Leinweber, A.W.Thomas and R.D. Young, Phys. Rev. D 69, 115006 (2004).

[19] V.F. Dmitriev and V.B. Telitsin Nucl Phys A 402581 (1983).

[20] I.S. Towner. Phys. Rep. 155, 263 (1987).

[21] V.V. Flambaum, A.Holl, C.D. Roberts and S.V. Wright, nucl-th/0510075 accepted to Few-Body Systems. A.Holl, P. Maris, C.D. Roberts and S.V. Wright, arXiv: nucl-th/0512048

[22] J. Dobaczevsky, J. Engel. Phys. Rev. Lett. 94, 232502 (2005).

[23] V.A. Dzuba, V.V. Flambaum, and M.V. Marchenko, Phys. Rev. A 68, 022506 (2003). 